\title{
El autoritarismo en la posguerra: un estudio de las actitudes de los salvadoreños
}

\author{
José Miguel Cruz'
}

\begin{abstract}
Resumen
Este artículo aborda el tema de las actitudes autoritarias de los salvadoreños. Para ello, el autor echa mano de una encuesta de opinión pública, realizada por el IUDOP en octubre de 1998, y utiliza una serie de ítemes sobre diversos temas en una escala para mostrar que gran parte de los salvadoreños tienen fuertes actitudes autoritarias. Estas tendencias actitudinales parecen ser más fuertes en los sectores más desaventajados socialmente, y podrían estar ligadas a la percepción del problema de la inseguridad ciudadana más que a otro problema percibido de la realidad.
\end{abstract}

\section{Introducción}

Hace siete años se firmaron los acuerdos de paz que pusieron fin a la guerra civil salvadoreña y, con ella, a la mayor parte de la violencia con motivaciones políticas. Los acuerdos no sólo suponían la finalización del conflicto, sino que también, y desde la perspectiva de la población, implicaron el inicio de la construcción de un modelo de sociedad que acabaría con los problemas fundamentales del país (ver Coleman, Cruz y Moore, 1996). Sin embargo, esto no parece haber sucedido así, al menos no como lo esperaba la mayor parte de la gente. De acuerdo con las encuestas de opinión pública, en 1995 y 1997, los salvadoreños se encontraban más decepcionados con respecto a la situación del país y pensaban que los principales problemas de éste - la clásica preocupación ciudadana por la economía y la violencia - eran aún más graves que en el pasado (IUDOP, 1995; IUDOP, 1997). De hecho, un sondeo realizado en agosto de 1997 reveló que uno de cada cuatro salvadoreños pensaba en emigrar del país.

El entusiasmo popular registrado por el fin de la guerra a inicios de la década dio lugar, en forma

1. Director del Instituto Universitario de Opinión Pública de la Universidad Centroamericana "José Simeón Cañas" (UCA). 
paulatina, a un ambiente de decepción relativamente generalizado, donde lo más sobresaliente era la visión negativa con respecto al país, a un pesimismo respecto al futuro del mismo y a un desencanto con las instituciones, sobre todo políticas, de la sociedad salvadoreña. La poca confianza en los políticos y órganos del Estado, la notable voluntad de emigrar del país y los elevados niveles de apatía ciudadana hacia cualquier forma de participación social y política, son sólo indicadores de un estado de ánimo poco esperanzador con respecto a la situación nacional. Las elecciones presidenciales de 1999 —aun a pesar de su dificultad para animar a la mayoría de la población en el evento nacional que es la elección del próximo gobierno nacional- han hecho olvidar de manera momentánea ese sentimiento de desánimo, o incluso de desesperación, que ha privado en buen parte de la sociedad salvadoreña, pero no lo han erradicado.

La gravedad de los problemas nacionales y el sentimiento de frustración social que prevalece en gran parte de la población salvadoreña compelen a muchos ciudadanos a hacer algo o, al menos, a idear formas de cambiar la situación. En estas condiciones, muchas veces los pensamientos sociales más comunes no siempre son los más adecuados desde una perspectiva de tolerancia democrática. Prueba de ello son los recurrentes llamados a establecer medidas como la pena de muerte, o propuestas de ley como la famosa "Ley de emergencia contra la delincuencia y el crimen organizado", en el entendido de que esto es lo que el país necesita para combatir la criminalidad.

En el caso salvadoreño, la percepción de un entorno social caótico y amenazante puede tener graves consecuencias para el desarrollo de la transición política, en la medida en que se comienzan a crear y articular respuestas psicosociales fundamentadas en actitudes antidemocráticas o autoritarias, que buscan responder de manera automática a ese contexto peligroso. Inglehart argumenta que una sociedad en la que no prevalecen la satisfacción por la vida (life satisfaction), satisfacción política (political satisfaction), confianza interpersonal (interpersonal trust) y apoyo para el orden social, es una sociedad menos propensa a adoptar y mantener instituciones democráticas (1988). El Salvador parece acercarse demasiado a esas condiciones. Otra encuesta del IUDOP, realizada en agosto de 1997, encontró que el 23 por ciento de los ciudadanos están poco o nada satisfechos con sus vidas personales, y cerca del 35 por ciento cree que el país va a estar peor dentro de cinco años. Por otro lado, los diversos sondeos de opinión pública muestran que los salvadoreños no se sienten representados políticamente y que confían muy poco en el sistema político (Cruz, 1997). Otros estudios han revelado que los salvadoreños $-y$, en general, los centroamericanos - tienen una visión negativa respecto a la confiabilidad de sus compatriotas (UNDP, 1998) Por último y de acuerdo también con otras investigaciones, la mayoría de los salvadoreños creen que el país no va por buen camino y que necesita un cambio; más aún, desde hace algunos años, alrededor del 30 por ciento de los ciudadanos sostienen que el país debe ser transformado en forma radical (IUDOP, 1994). Todos estos datos muestran que no se cuentan con las condiciones más favorables.

Uno de los principales generadores de ansiedad ciudadana y que parece estar vinculado muy estrechamente a esa percepción desconcertante de la realidad salvadoreña es la violencia delincuencial. Una investigación del Instituto Universitario de Opinión Pública (1998a) encontró que los ciudadanos salvadoreños están ahora mucho más preocupados por la violencia de lo que estaban en años anteriores, ello a pesar de que la misma pesquisa revela que los porcentajes de victimización por la violencia se habrían reducido en los últimos dos años; más aún, dicho estudio encontró que "casi el 80 por ciento de los encuestados vive con niveles altos de ansiedad a causa de la delincuencia". Estos niveles de angustia social no sólo tienen repercusiones en el comportamiento cotidiano de las personas, limitan su quehacer diario o estimulan medidas personales que buscan evitar el peligro, sino que también pueden tener repercusiones más amplias, que alcanzan lo político y el acuerdo tácito de convivencia social.

Ahora bien, como ha sido señalado por algunos autores (Agüero, 1998; Holston y Caldeira, 1998), el control sobre la violencia y sobre la institucionalización de la misma en sociedades en transición, representa uno de los problemas que pueden amenazar los procesos de transición democrática, sobre todo en sus etapas de consolidación (.O'Donnell, 1994; Valenzuela, 1992). En el caso salvadoreño, esta amenaza desde el fenómeno de la violencia no parece venir tanto desde el ejercicio de la misma por parte del Estado, al menos no como en el pasado, sino - y a diferencia de países 
como Brasil-, desde la configuración de un sistema actitudinal y normativo en la cultura política como respuesta a la percepción de inseguridad que genera la violencia criminal y social en la actualidad. Esta respuesta cultural y psicosocial se basa en la adquisición de actitudes de corte autoritario que buscan responder eficaz y rápidamente al problema de la anarquía generada, en particular, por la criminalidad.

Este pequeño artículo pretende iniciar el abordaje de este fenómeno que podría estar tomando lugar en la sociedad salvadoreña — si no tiene ya larga data de presencia-; es decir, se exploran las posibles actitudes autoritarias que podrían estar tomando lugar como respuesta a esta desesperación por la situación del país. El artículo se basa en un sondeo de opinión pública llevado a cabo por el Instituto Universitario de Opinión Pública, en octubre de 1998, el cual recoge las actitudes de los ciudadanos respecto a los aspectos siguientes: la necesidad de contar con una personalidad autoritaria, el irrespeto por la legali-
De acuerdo con Altemeyer (1996), el autoritarismo se puede identificar por la existencia de tres tendencias actitudinales: $(a)$ una sumisión a la autoridad; (b) una aprobación a la agresión autoritaria; y, (c) convencionalismo. ámbito de estudio de diversas disciplinas y en cada una la concepción sobre el mismo puede ser muy diferente, o puede llevar a visiones distintas. En realidad, y por cuestiones de espacio e importancia sobre el tema por desarrollar, no es la intención de este trabajo abordar ese dilema. Baste decir que el tema del autoritarismo será abordado desde su concepción psicosocial, no política, la cual se centrará en revisar actitudes más que estructuras de personalidad.

El estudio del autoritarismo en el ámbito de la psicología social fue claramente marcado hace ya medio siglo por un grupo de académicos de Berkeley, liderados por Theodor Adorno. Ellos definieron un tipo de personalidad a la cual llamaron autoritaria, la cual posibilitaba o estimulaba la creación o el surgimiento de regímenes como el nazismo. La idea central era que una sociedad en la que imperaba ese tipo de personalidad era más propensa a crear un Estado violento. Esta tesis ha sido ampliamente discutida y puesta en duda, pero en lo fundamental ha sido dad del país, la inclinación extrema por el orden social, la vocación por el uso de medidas drásticas y la intolerancia.

Es necesario decir que este escrito constituye el primer paso de una serie de trabajos que tienen como objeto estudiar las implicaciones de una sociedad que se ve abrumada por los problemas sociales, en especial por la violencia, en la construcción de actitudes y normas autoritarias que pueden llegar a estimular -o al menos justificar - el reaparecimiento de figuras autoritarias en el ámbito político salvadoreño. Por el momento, el objetivo de este artículo es describir aquéllas actitudes de la población que son la base para la formación de esquemas autoritarios de pensamiento. En los subsiguientes trabajos se efectuarán los ejercicios de vinculación analítica con otros elementos de la realidad salvadoreña.

\section{Las actitudes autoritarias}

Definir autoritarismo es siempre un problema, no sólo por las diversas acepciones que suelen dársele, sino porque el concepto en sí mismo es muy útil para el estudio de las actitudes vinculadas con el autoritarismo (Moreno Martín, 1997).

De acuerdo con Altemeyer (1996), el autoritarismo se puede identificar por la existencia de tres tendencias actitudinales: (a) una sumisión a la autoridad; (b) una aprobación a la agresión autoritaria; y, (c) convencionalismo. La sumisión a la autoridad implica una disposición a respetar y obedecer a las autoridades sin ninguna objeción, pero esto no significa hacerlo con todas las autoridades, sino con aquéllas que están más cerca de las fuentes de autoridad fundamentales: Dios, el líder máximo o el orden social. Por su parte, la agresión autoritaria no necesariamente implica ser violento, pero sí implica la aprobación del uso de la violencia para respetar la autoridad y mantener a toda costa el orden social, o lo que está prescrito. Finalmente, como convencionalismo se entiende una fuerte aceptación y compromiso con las normas tradicionales de una sociedad, tales normas no implican de manera necesaria el marco jurídico formal de la sociedad, sino las "leyes divinas", lo que viene de la Biblia y lo que constituye la palabra de Dios. 


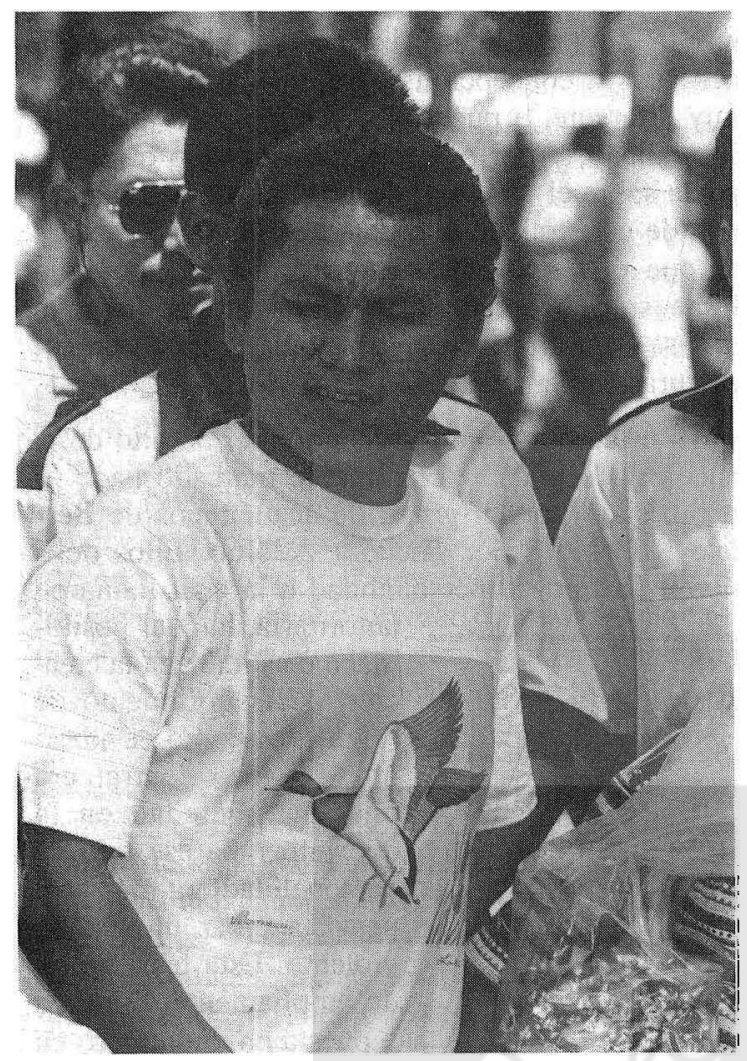

Otros han entendido autoritarismo esencialmente con los ingredientes de adhesión al principio de autoridad y la inclinación a la uniformidad en las personas, es decir, el rechazo a la diversidad (Moreno Martín, ídem).

Como ya se mencionó, en este trabajo se utilizarán algunos ítems que exploran algunos aspectos que pueden estar relacionados con lo anterior: intolerancia, adhesión al orden social, justificación de medidas drásticas y violencia, y la simpatía por una personalidad autoritaria.

\section{Aspectos metodológicos}

El presente artículo se basa en los resultados de una pesquisa de opinión pública realizada por el Instituto Universitario de Opinión Pública, a mediados del mes de octubre de 1998. La encuesta en sí misma tenía como objeto conocer algunos aspectos de la cultura política salvadoreña, así como también las expectativas de los ciudadanos hacia la política y sus actores; indagar sobre el interés y la confianza que tiene la población en la política nacional y, a la vez, recoger las preferen- cias partidistas de cara a las elecciones presidenciales que se llevarán a cabo en 1999.

\subsection{El cuestionario y la escala de autoritarismo}

Lo que sigue a continuación es una descripción metodológica del sondeo en cuestión. No está de más añadir que este trabajo se concentra en apenas una pequeña parte de los resultados obtenidos en esa encuesta, resultados que tienen relación con una batería de ocho ítemes al estilo de escala de Likert, que exploraban actitudes en torno al autoritarismo. El cuestionario empleado para llevar a cabo la investigación se componía básicamente de siete partes. En la primera se recogían los datos sociodemográficos del encuestado; la segunda parte del cuestionario estaba constituida por una serie de preguntas que exploraban las actitudes de las personas encuestadas relacionadas con la cultura política del país, y dentro de las cuales están incluidos los reactivos de interés para este artículo; en la tercera sección se recopilaban las expectativas que tiene la población hacia la política y los futuros gobernantes del país, e hicieron referencia al ganador de las elecciones de marzo de 1999; el cuarto apartado de la encuesta estaba dirigido a recoger la confianza que la población tiene en diferentes instituciones políticas del país; la quinta sección estaba diseñada a sondear la participación política y electoral de los ciudadanos; mientras que la última parte del cuestionario agrupaba preguntas que exploraban la percepción de los encuestados sobre el proceso electoral del 1999 (opiniones políticas y preferencias de los partidos).

Los reactivos utilizados en el presente trabajo son los siguientes y se pueden dividir según los distintos tópicos. Dos ítemes exploraban la simpatía de la población por una personalidad autoritaria:

- Lo que El Salvador necesita es un hombre fuerte y decidido que ponga orden.

- Los políticos que ganen las elecciones deben ser como el general Martínez.

La inclinación extrema por el orden social fue medida por dos ítemes:

- Las manifestaciones sociales sólo consiguen armar alboroto y crear problemas sociales y políticos.

- En lugar de derechos humanos, lo que nuestra sociedad necesita es mucha ley y orden. 
La vocación por medidas drásticas se recogía a través de los siguientes reactivos:

- La pena de muerte es necesaria para combatir la delincuencia en el país.

- Los derechos humanos favorecen a los delincuentes y asi no se puede acabar con ellos.

- La situación de nuestro país está tan mal que la única forma de sacar adelante a nuestro país es eliminar con mano dura a los que causen problemas.

Y finalmente un ítem medía intolerancia:

- La sociedad no debería aguantar a aquéllos que tienen ideas políticas que son extremadamente diferentes de la mayoría.

Todos estos reactivos tenían cinco opciones de respuesta: muy de acuerdo, algo de acuerdo, indeciso, algo en desacuerdo y muy en desacuerdo. La gente, los encuestados, escogían la opción que mejor representara su posición ante el ítem en cuestión.

Estos ítemes formaron una escala que se denominó "Escala de autoritarismo", con la cual fue posible medir las tendencias autoritarias de los salvadoreños. La misma fue resultado de la sumatoria de las respuestas de todos los ítemes anteriores y tiene un buen nivel de confiabilidad, según la prueba Alfa de Cronbach (0.725). Esta escala tiene un rango que va desde el valor 8 hasta el valor $40^{2}$, pero para efectos prácticos y hacerlo más comprensible al lector, lo convertimos a un rango del 1 al 10 , donde 1 representa el menor nivel de autoritarismo y 10 significa el mayor nivel de actitud autoritaria.

\subsection{Muestra y procedimiento}

La muestra nacional de la encuesta se diseñó de tal manera que reflejase lo más fielmente posible la totalidad de la población salvadoreña, según los datos del Censo Nacional de Población de 1992 y del informe de la Encuesta de Hogares de Propósitos Múltiples, de la Dirección de Información del Ministerio de Economía de 1996. La cuota muestral incluyó 34 municipios de los catorce departamentos de la República. En cada departamento, la muestra se dividió en dos grandes secto- res: urbano y rural. El sector rural se dejó como una categoría aparte por las dificultades de encontrar criterios de tipificación en el campo. En el muestreo se consideraron también cuotas por sexo y edad de los encuestados, de tal manera que cada boleta estaba marcada con las características que debía cumplir el encuestado para ser considerado en la investigación.

La aplicación del cuestionario se hizo por aproximación no sistemática a los hogares ubicados en los municipios y las zonas definidas en forma aleatoria. Los entrevistadores explicaban a las personas abordadas los objetivos y el tema de la encuesta. En cada caso se entrevistó sólo a personas que quisieran contestar (una persona por hogar), y que cumplieran con los requerimientos descritos en la boleta para completar la cuota muestral.

La muestra final obtenida fue de 1,247 encuestas válidas, con un margen de error estimado de +1 - 0.04 (cuatro por ciento). El 48.7 por ciento de los encuestados pertenecía al sexo masculino y el 51.3 por ciento, al sexo femenino. La edad promedio fue de 36.2 de años, con una desviación típica de 14.39 años. Los entrevistados tenían un promedio general de 8.13 años de estudio, y el 51.6 por ciento se encontraba empleado en la actualidad o poseía un trabajo propio.

\section{Los resultados}

En el Cuadro 2 se presentan los resultados porcentuales de cada uno de los ítems que forman la escala. Como puede verse, en términos generales parece haber una distribución que favorece más el apoyo de actitudes autoritarias que el rechazo a las mismas. En el tema de la figura autoritaria, la gran mayoría de salvadoreños, el 86.6 por ciento, expresaron que el país necesita un líder fuerte y decidido que ponga orden, mientras que sólo el 11.3 por ciento rechazó esa actitud. Sin embargo, podría argumentarse que una personalidad "fuerte y decidida que ponga orden" no es sinónimo de autoritario, eso es cierto y, por tanto, hay que considerar que mucha gente pide simplemente firmeza y no autoritarismo. En tal sentido, el ítem que pregunta por el general Martínez puede ser más preciso en el tema de autoritarismo; en este caso, la figura - ya mítica- del general es la de una persona que

2. Las respuestas en cada ítem tenían también un valor numérico. "Muy en desacuerdo" era igual a 1, algo en desacuerdo tenía un valor de 2 , y así sucesivamente hasta "muy de acuerdo", que tenía el valor de 5 . La sumatoria de los ocho ítemes podía proporcionar un puntaje mínimo de 8 y un máximo de 40. 
Cuadro 1
Distribución de la población encuestada según departamento y sector social

\begin{tabular}{|c|c|c|c|c|c|c|c|c|c|}
\hline \multirow[b]{2}{*}{ Departamento } & & \multirow[b]{2}{*}{ Alto } & \multirow[b]{2}{*}{ Medio-alto } & \multicolumn{2}{|c|}{ Sector social } & \multirow[b]{2}{*}{ Marginal } & \multicolumn{2}{|c|}{ Todos } & \multirow[b]{2}{*}{$\%$} \\
\hline & & & & Medio-bajo & Obrero & & Rural & $\mathrm{N}$ & \\
\hline \multirow[t]{2}{*}{ Todos } & $\%$ & 7.2 & 12.8 & 13.3 & 20.1 & 13.2 & 33.4 & - & 100.0 \\
\hline & $N$ & 90 & 159 & 166 & $25 I$ & 164 & 417 & 1247 & - \\
\hline Ahuachapán & & 3 & 4 & 4 & 8 & 4 & 42 & 65 & 5.2 \\
\hline Santa Ana & & 8 & 10 & 16 & 20 & 13 & 45 & 112 & 9.0 \\
\hline Sonsonate & & 6 & 10 & 10 & 16 & 10 & 35 & 87 & 7.0 \\
\hline La Libertad & & 8 & 16 & 16 & 25 & 15 & 50 & 130 & 10.4 \\
\hline Chalatenango & & 3 & 4 & 4 & 8 & 4 & 21 & 44 & 3.5 \\
\hline San Salvador & & 36 & 62 & 63 & 93 & 63 & 48 & 365 & 29.3 \\
\hline Cabañas & & 2 & 3 & 3 & 6 & 4 & 16 & 34 & 2.7 \\
\hline Cuscatlán & & 2 & 5 & 5 & 8 & 4 & 15 & 39 & 3.1 \\
\hline San Vicente & & 2 & 4 & 4 & 6 & 6 & 13 & 35 & 2.8 \\
\hline La Paz & & 3 & 8 & 8 & 12 & 8 & 20 & 59 & 4.7 \\
\hline Usulután & & 4 & 8 & 8 & 12 & 8 & 38 & 78 & 6.3 \\
\hline San Miguel & & 8 & 15 & 15 & 21 & 15 & 21 & 95 & 7.6 \\
\hline Morazán & & 1 & 4 & 4 & 6 & 4 & 24 & 43 & 3.4 \\
\hline La Unión & & 4 & 6 & 6 & 10 & 6 & 29 & 61 & 4.9 \\
\hline
\end{tabular}

cumple con las características del autoritarismo, fanático del orden, capaz de usar al extremo la violencia para mantenerlo ${ }^{3}$ y de marcada intolerancia a la disensión; la ventaja de esta personalidad es que es ampliamente conocida por todos los salvadoreños, aun por los jóvenes, a quienes se les han transmitido esos rasgos de la imagen. Este ítem mostró una división más grande de las opiniones: el 44.1 por ciento estuvo de acuerdo con que los políticos sean como Martínez, mientras que el 32.3 por ciento mostró su rechazo; además, el 23.7 por ciento se mostró indeciso frente a este reactivo. Con todo, los resultados muestran un porcentaje
Ahora bien, los reactivos que mostraron una disposición al uso de medidas drásticas y agresivas como una manera de solucionar problemas o como forma de castigo, presentaron un amplio apoyo entre la población. mayor de gente que apoya la idea de un político autoritario - como el general Hernández Martínez- en lugar de rechazarla.

3. Para el caso, son muy ilustrativas las anécdotas que populamente se conocen del dictador. Las más famosas se refieren a que los delincuentes eran castigados cortándoles las manos o ahorcándolos en público.

4. En realidad, fue una de las que tuvieron más alta correlación con el total de ítemes de la escala y que contribuyó, en forma significativa, al nivel de confiabilidad de la misma. 


\section{Cuadro 2 \\ Actitudes relacionadas con el autoritarismo \\ (En porcentajes)}

\begin{tabular}{|c|c|c|c|c|c|}
\hline \multirow[b]{2}{*}{ ACTITUD } & \multirow[b]{2}{*}{$\begin{array}{c}\text { Muy en } \\
\text { desacuerdo }\end{array}$} & \multicolumn{3}{|c|}{ RESPUESTA } & \multirow[b]{2}{*}{$\begin{array}{l}\text { Muy de } \\
\text { acuerdo }\end{array}$} \\
\hline & & $\begin{array}{c}\text { Algo en } \\
\text { desacuerdo }\end{array}$ & Indeciso & $\begin{array}{l}\text { Algo de } \\
\text { acuerdo }\end{array}$ & \\
\hline $\begin{array}{l}\text { Lo que El Salvador necesita es un hombre fuerte y } \\
\text { decidido que ponga orden. }\end{array}$ & 6.7 & 4.6 & 2.1 & 17.6 & 69 \\
\hline $\begin{array}{l}\text { Los políticos que ganen las elecciones deberían ser } \\
\text { como el general Martínez. }\end{array}$ & 25.2 & 7.1 & 23.7 & 16.3 & 27.8 \\
\hline $\begin{array}{l}\text { La situación de nuestro país está tan mal que la única } \\
\text { manera de sacarlo adelante es eliminar con mano } \\
\text { dura a los que causen problemas. }\end{array}$ & 13.5 & 7.8 & 3.6 & 18.2 & 56.9 \\
\hline $\begin{array}{l}\text { Los derechos humanos favorecen a los delincuentes y } \\
\text { así no se puede acabar con ellos. }\end{array}$ & 10.4 & 8.1 & 6.2 & 21.1 & 54.2 \\
\hline $\begin{array}{l}\text { La pena de muerte es necesaria para combatir la } \\
\text { delincuencia en el país. }\end{array}$ & 29.9 & 6.9 & 5.1 & 15.1 & 43.1 \\
\hline $\begin{array}{l}\text { En lugar de derechos humanos lo que nuestro país } \\
\text { necesita es mucha ley y orden. }\end{array}$ & 6.4 & 5.8 & 2.7 & 20.3 & 64.8 \\
\hline $\begin{array}{l}\text { Las manifestaciones callejeras sólo consiguen } \\
\text { armar alboroto y crear problemas sociales y políticos. } \\
\text { La sociedad no debería aguantar a aquéllos } \\
\text { que tienen ideas políticas que son extremadamente } \\
\text { diferentes a las de la mayoría. }\end{array}$ & 11.4 & 12.6 & 12.7 & 22.0 & 52.8 \\
\hline
\end{tabular}

miento muy drástico, tuvo una amplia aceptación entre los ciudadanos salvadoreños: más del 56 por ciento dijo estar muy de acuerdo con la misma y alrededor del 18 por ciento estuvo algo de acuerdo; sólo el 21.3 por ciento rechazó esa afirmación. En proporciones similares se distribuyeron los encuestados con respecto al ítem de que los derechos humanos favorecían a los delincuentes y, por tanto, no se les podía combatir. Esto significa no sólo que la gente quiere medidas más drásticas, sino que la mayoría estaría dispuesta a prescindir de la protección de los derechos humanos con tal de enfrentar el problema de la delincuencia que los tiene agobiados. Este tipo de respuesta parece ir en la misma línea de las que muestran que muchos ciudadanos no valoran la importancia de los derechos humanos en una sociedad como la salvadoreña (ver Cano y Rodríguez, 1996). Finalmente, el 58.2 por ciento de los consultados apoyó la pena de muerte; este dato no es nuevo, desde hace varios años gran parte de los salvadoreños insisten en la reinstalación de esa medida como una forma para combatir la delincuencia en el país.
La inclinación por el orden social se midió de dos formas. En primer lugar, se preguntó directamente por la necesidad de la ley y el orden y, en segundo lugar, se interrogó sobre las manifestaciones callejeras como un posible generador de desorden social. De acuerdo con los resultados, más del 80 por ciento reivindicó la ley y el orden por encima del respeto a los derechos humanos, inclusive sólo el 12 por ciento se mantuvo en contra de ese pensamiento. Por otro lado y con la misma tendencia, la mayoría de los salvadoreños no ven de buen agrado las manifestaciones callejeras: casi el 75 por ciento compartió la opinión de que tales manifestaciones sólo consiguen armar alboroto y problemas sociales. Ello a pesar de la historia de movilización ciudadana que ha caracterizado al país en el pasado; sin embargo, parece que los ciudadanos no están más dispuestos - ¿será porque están cansados? - a consentir ese tipo de expresiones públicas.

Por último, el único reactivo que midió intolerancia arrojó resultados un poco más divididos que los anteriores. Un poco más de la mitad de los consultados estuvo de acuerdo con la idea de que 
la sociedad no debería aguantar a aquéllos que tienen ideas políticas que son extremadamente diferentes de los de la mayoria; mientras que una tercera parte de la gente estuvo en desacuerdo con esa opinión.

A simple vista, todos estos resultados sugieren que los ciudadanos salvadoreños están más dispuestos a apoyar actitudes y opiniones autoritarias, que a no apoyarlas o rechazarlas. Aspectos como la justificación de medidas drásticas y la proclividad por valorar el orden por sobre otros valores como los derechos humanos, dicen mucho acerca de las actitudes sociales de los salvadoreños y de su disposición para apoyar respuestas sociales autoritarias.

Ahora bien, alguien podría argumentar como respuesta a estas cifras que éstas reflejan opiniones aisladas y que, en realidad, tales actitudes están más condicionadas por una realidad abrumante que por una convicción personal de los ciudadanos. Ciertamente, este tipo de respuestas sociales no pueden aislarse del contexto en el cual se expresan, pero tampoco puede descartarse un factor individual que hace que tal tipo de pensamientos tenga más cabida que otros como respuesta a la percepción de la realidad. Así como tampoco pue- de decirse que estas opiniones aparecen de forma aislada y sin relación entre ellas. Los mismos resultados muestran que todos estos ítemes que forman la escala están vinculados entre ellos; es decir, las personas que expresaron su compromiso con un tipo de pensamiento, en buena medida lo hicieron también con los demás. Ello puede verse con más claridad en el Cuadro 3.

El cuadro en cuestión contiene una matriz de correlaciones de Pearson de los ocho ítemes que constituyen la escala de autoritarismo, para mostrar los niveles de asociación entre los mismos. Los resultados indican que no existe ningún ítem que no tenga algún nivel de correlación significativo $(p<0.001)$ con otro. Es decir, todos los reactivos de la escala están relacionados entre sí con validez estadística; no obstante, el nivel de asociación varía y no es el mismo para todas las combinaciones posibles. Por ejemplo, el coeficiente más alto de correlación $(r=0.3779)$ se encuentra en los ítemes $C$ (La situación de nuestro país está tan mal que la única manera de sacarlo adelante es eliminar con mano dura a los que causen problemas) y $F$ (La pena de muerte es necesaria para combatir la delincuencia en el país), lo que significa que varias personas que están de acuerdo con eliminar a quienes causen problemas, también es-

Cuadro 3

Matriz de correlación de los ítemes de la escala de autoritarismo

\begin{tabular}{|c|c|c|c|c|c|c|c|}
\hline ACTITUDES & A & B & $\mathrm{C}$ & $\begin{array}{c}\text { ACTITUDES } \\
\text { D }\end{array}$ & $\mathrm{E}$ & F & G \\
\hline B & $0.2783^{*}$ & & & & & & \\
\hline $\mathrm{C}$ & $0.3746^{*}$ & $0.3008 *$ & & & & & \\
\hline D & $0.2399 *$ & $0.2641^{*}$ & $0.3381^{*}$ & & & & \\
\hline $\mathrm{E}$ & $0.2222 *$ & $0.2939^{*}$ & $0.3477 *$ & $0.2397 *$ & & & \\
\hline $\mathrm{F}$ & $0.3584 *$ & $0.2473 *$ & $0.3779 *$ & $0.3036 *$ & $0.2393 *$ & & \\
\hline G & $0.2539 *$ & $0.1520^{*}$ & $0.2360^{*}$ & $0.3356^{*}$ & $0.2039 *$ & $0.2495^{*}$ & \\
\hline $\mathrm{H}$ & $0.1699^{*}$ & $0.2186^{*}$ & $0.2827^{*}$ & $0.1841 *$ & $0.1707^{*}$ & $0.2049 *$ & $0.1355^{*}$ \\
\hline
\end{tabular}

* $p<0.001$

(A) Lo que El Salvador necesita es un hombre fuerte y decidido que ponga orden. (B) Los políticos que ganen las elecciones deberían ser como el general Martínez. (C) La situación de nuestro país está tan mal que la única manera de sacarlo adelante es eliminar con mano dura a los que causen problemas. (D) Los derechos humanos favorecen a los delincuentes y así no se puede acabar con ellos. (E) La pena de muerte es necesaria para combatir la delincuencia en el país. (F) En lugar de derechos humanos lo que nuestro país necesita en realidad es mucha ley y orden. (G) Las manifestaciones callejeras sólo consiguen armar alboroto y crear problemas sociales y políticos. $(\mathrm{H})$ La sociedad no debería aguantar a aquéllos que tienen ideas políticas que son extremadamente diferentes de la mayoría. 
tán de acuerdo con la pena de muerte como medida para combatir la delincuencia. Algo parecido sucede al ver el coeficiente de correlación entre los ítemes $A$ y $C(\mathrm{r}=0.3746)$, lo cual señala que la percepción de la necesidad de mano dura suele ir acompañada del deseo de la presencia de un hombre fuerte y decidido que ponga orden, y este deseo, a su vez, va en la misma dirección de apoyar la pena de muerte $(r=0.3584)$.

Otras correlaciones destacables se encuentran entre la necesidad de eliminar con mano dura a los causantes de los problemas, y la concepción de los derechos humanos como protectores de delincuentes $(r=0.3381)$, y entre éste y la oposición hacia las manifestaciones callejeras ( $r=0.3356)$. Al final, todos los ítemes están correlacionados entre sí, lo cual indica cómo las diversas actitudes están ancladas unas con otras y algunas más que otras. $\mathbf{L a}$ actitud que parece separarse un poco de las demás, aunque sin desvincularse, es la de la intolerancia; sus correlaciones con el resto de actitudes poseen los coeficientes más bajos, aunque — se insiste- no dejan de ser significativos estadísticamente.

Todo lo anterior muestra, por tanto, que esas actitudes, la simpatía por figuras fuertes, la inclinación por mantener el orden, la aprobación de la pena de muerte, la justificación de la eliminación de otras personas, el rechazo hacia los derechos humanos y la intolerancia, son elementos o factores de una misma inclinación actitudinal, la del autoritarismo.

Ahora bien, ¿qué tanta inclinación por el autoritarismo tienen los salvadoreños? La respuesta viene dada por los resultados de la escala creada a partir de los ítemes anteriores. Sólo es necesario recordar, en este espacio, que la escala es el producto de la sumatoria de todos los ítemes, de tal manera que el puntaje mayor " 10 " significa que una persona contestó "muy de acuerdo" a todas las preguntas planteadas, en tanto que el puntaje menor "1" significa que la persona respondió "muy en desacuerdo". Dado que la mayor parte de las personas no responden de esa forma tan alineada, la mayor parte de puntajes están entre esos dos valores, y su distribución a lo largo de esa escala es útil para ver hacia dónde se orientan los salvadoreños. Los resultados de la escala se presentan en la Figura 1.
Figura 1

Distribución de los puntajes en la escala de actitudes autoritarias

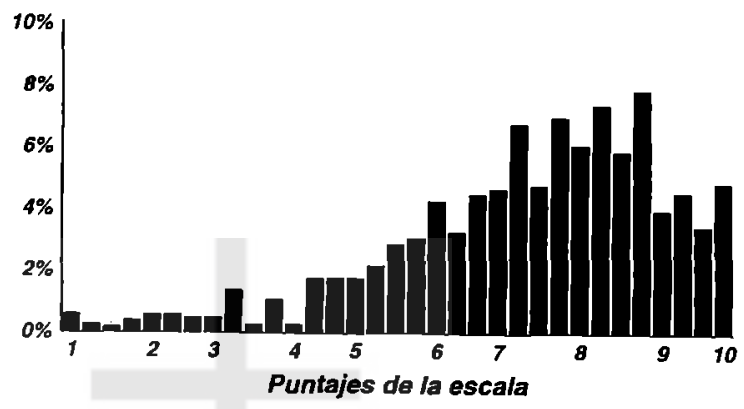

Como puede verse, la mayor parte de los salvadoreños se ubica en los valores más altos de la escala de autoritarismo. El 50 por ciento de los consultados se encuentra entre los valores 7.7 y 10 , lo que significa que más de la mitad de la población tiene fuertes actitudes autoritarias en El Salvador. Por el contrario, menos del 25 por ciento de los encuestados se ubican por debajo del valor 5 , es decir, con bajos niveles de actitudes autoritarias. ¿Significa, lo anterior, que los salvadoreños son autoritarios? Quizá la mejor pregunta no sea si son autoritarios o no, sino si tienen actitudes autoritarias; dado que para calificar de esa forma a los ciudadanos se tendría que esta hablando de estructuras de la personalidad al estilo planteado por Adorno y sus colaboradores (ídem.). Lo cierto es que este estudio se refiere a actitudes, y puede ser que éstas sean parte de una forma cultural de respuesta ante la realidad salvadoreña plagada de violencia y desigualdad; pero hasta donde muestran los datos, hay que reconocer que los ciudadanos salvadoreños están más ínclinados por el autoritarismo que por lo opuesto.

No obstante y como ya puede sugerirlo la misma figura, no todos los ciudadanos tienen esa inclinación por el autoritarismo de la misma manera. Algunas personas pueden tener más esa orientación que otras $y$, por lo tanto, es importante identificar las variables que pueden hacer esa diferencia. De acuerdo con las cifras presentadas en el Cuadro 4, las actitudes autoritarias varían en función de todas las variables sociodemográficas estudia- 


\section{Cuadro 4}

Promedios en la escala de actitudes autoritarias según variables sociodemográficas

\begin{tabular}{lc}
\hline Variables & Promedio \\
\hline Todos & 7.31 \\
Sexo * & \\
Masculino & 7.09 \\
Femenino & 7.51 \\
& \\
Edad ${ }^{*}$ & \\
18 a 25 años & 7.66 \\
26 a 40 años & 7.22 \\
41 a 55 años & 6.85 \\
56 años y más & 7.48 \\
& \\
& \\
Estrato * & \\
Alto & \\
Medio-alto & 7.00 \\
Medio-bajo & 6.39 \\
Obrero & 7.10 \\
Marginal & 7.50 \\
Rural & 7.52 \\
& 7.60 \\
Educación * & \\
Ninguna & \\
Primaria & 7.76 \\
Básica & 7.62 \\
Bachillerato & 7.57 \\
Superior & 7.58 \\
\hline a & 6.23 \\
\hline arior &
\end{tabular}

$*^{*} \mathrm{p}<0.001$

das, esto es, sexo, edad, estrato socioeconómico y nivel de estudios 5 .

El promedio general para toda la población fue de 7.31, lo que sugiere que, como ya se había adelantado, los salvadoreños tienden a estar más inclinados hacia actitudes autoritarias. Ahora bien, dentro de esa corriente hay matices. Las mujeres obtienen un promedio levemente más alto que los hombres en la escala, lo que significa que tienden a respaldar un poco más actitudes autoritarias que las personas del sexo opuesto. En el caso de la edad, el grupo que más destaca es el de los jóvenes entre 18 y 25 años. Estos apoyan más medidas drásticas, simpatizan más con figuras autoritarias y están más dispuestos a privilegiar el orden social que el resto de sus compatriotas; por el contrario, las personas que puntúan más bajo en la escala -aunque eso no los hace rechazar la tendencia- son los adultos entre 41 y 55 años de edad, es decir, los adultos que ocupan puestos decisivos en la sociedad.

Pero las variables que separan más las tendencias actitudinales de los ciudadanos son las de carácter social: el estrato socioeconómico y la educación. Las personas de los estratos populares (obreros, marginales y campesinos) parecen ser los más dispuestos a cumplir con las actitudes de autoritarismo que mide la escala; en cambio, esos niveles son menores entre las personas de clases mediaalta y alta, pero especialmente entre las personas de clase media-alta; éstos se separan en forma significativa del resto de ciudadanos y tienen una tendencia menor a poseer inclinaciones autoritarias. Asimismo, las actitudes autoritarias parecen estar fuertemente vinculadas con el nivel de educación de la persona; en la medida en que el ciudadano dispone de mayor formación educativa, en esa medida su propensión al autoritarismo se reduce. Las personas con instrucción superior son los que están menos dispuestos a apoyar medidas drásticas, a pedir personalidades autoritarias y a ser intolerantes. De las dos variables, la educación parece ser la que más determina esos cambios.

Lo anterior pone de relieve, sin duda, la función de las condiciones sociales de las personas a la hora de formar y mantener sus actitudes. Una persona en desventaja social, que vive en la pobreza, sin mayor formación educativa y con nada más que su fuerza de trabajo para sobrevivir, quizá estará más dispuesta a respaldar medidas como la pena de muerte, exigir políticos autoritarios, justificar medidas de agresión con tal de mantener el orden, rechazar la utilidad de los derechos humanos y ser más intolerante frente al disenso, que aquéllas personas con mejores condiciones de vida.

La percepción de que las cosas están mal y de ahí la necesidad de solucionarla apelando a las soluciones más drásticas viene, por tanto, en buena

5. Esto según la pruebas estadísticas. Para el caso, se utilizó el Análisis de varianza de Kruskal-Wallis, una prueba no paramétrica; ello porque las varianzas entre los diversos grupos no eran homogéneas. 
medida de la vivencia cotidiana de la gente, de su falta de recursos para enfrentar de manera eficaz los problemas más urgentes que ponen en peligro su supervivencia. De ahí que, probablemente, estas actitudes sociales que parecen tener un amplio apoyo entre los salvadoreños más pobres, más desaventajados, no sean más que la respuesta particular frente a unas circunstancias anormales, en el sentido ético de la palabra. El apoyo a las medidas drásticas, la pena de muerte y las figuras autoritarias viene como resultado de la percepción de que las cosas no han podido resolverse, sobre todo en el campo de la violencia y la inseguridad pública.

De hecho, los resultados muestran que las personas que se encuentran más preocupadas por la violencia criminal, como problema fundamental del país, son aquéllas que tienen más inclinación de tipo autoritario (7.58, en la escala usada); en cambio, los ciudadanos preocupados por otros problemas como la gobernabilidad del país, el medio ambiente, la corrupción, etcétera, poseen menos inclinación por el autoritarismo que el resto (6.30).

\section{Resumen}

Con este trabajo se pretende iniciar el estudio de las actitudes autoritarias de la población salvadoreña en la posguerra, y sus implicaciones para el desarrollo de la transición política. El presente artículo ha presentado, en términos generales y teóricos, la importancia de estudiar las actitudes autoritarias de lá población salvadoreña que parecen estar tomando auge en los últimos años. Sin embargo, en su contenido fundamental se ha hecho una descripción de cómo diversas actitudes que reclaman más dureza, menos aprecio por lo derechos humanos, más preocupación por mantener el orden social y la intolerancia, entre otras cosas, parecen estar vinculadas entre sí en una gran tendencia de pensamiento social que alcanza a más del 50 por ciento de la población, sobre todo a la más desaventajada socialmente, la más pobre y marginada.
Este artículo no ha pretendido establecer que los salvadoreños son autoritarios o que tengan una personalidad autoritaria, sino que muchas de sus actitudes, que determinan parte de su comportamiento cotidiano, o al menos de su visión de la realidad o de lo que debe ser la realidad, tienen una inclinación hacia el autoritarismo, que no puede ser soslayada a la hora de buscar explicaciones a la conducta social de los salvadoreños. Por el momento, estos resultados generan más preguntas que respuestas. ¿Será que detrás de esas actitudes se esconde una forma de ser claramente autoritaria? ¿Se puede decir, entonces, que la mayoría de los ciudadanos son autoritarios? ¿Acaso la violencia social que embarga al país podría explicar en gran medida ese tipo de actitudes que prevalece en buena parte de los salvadoreños? ¿Cómo se han generado semejantes actitudes y qué las alimenta? ¿Qué impacto podría tener en la sociedad salvadoreña el que estas actitudes comenzaran a traducirse por completo en comportamientos en la misma magnitud? ¿Qué se podría hacer para revertir estas tendencias de pensamiento?

Todas estas interrogantes esperan ser abordadas en posteriores trabajos. Por ahora, estos son los resultados que merecen discusión.

\section{Bibliografía}

Adorno, Theodor W., et al., La personalidad autoritaria, Buenos Aires: Proyección, 1965.

Agüero, Felipe, "Conflicting assessments of democracy: exploring the fault lines", En Felipe Aguiero y Jeffrey Stark (eds.), Fault lines of democracyin posttransition Latin America, Miami: North-South Center Press, 1998.

Altemeyer, Bob, The authoritarian specter, Cambridge: Harvard University Press, 1996.

Cano, Ignacio y Rodríguez Loza, Carolina, La representación social de los derechos humanos en El Salvador, Realidad, 50, 1996, pp. 307-350.

Coleman, Kenneth M.; Cruz, José Miguel y Moore, Peter J., "Retos para consolidar la democracia en El 
Salvador", Estudios Centroamericanos (ECA), 57I572, 1996, pp. 415-440.

Cruz, José Miguel, "Elecciones y pensamiento social: opinión pública en los comicios 97", en Héctor Dada (ed.), Las elecciones de 1997: ¿un paso más en la transición democrática?, San Salvador: FLACSO, 1997.

Instituto Universitario de Opinión Pública (IUDOP), Encuesta de opinión sobre delincuencia, Serie de informes 70, San Salvador: IUDOP-UCA, 1998a.

Instituto Universitario de Opinión Pública (IUDOP), Encuesta sobre cultura política, Serie de informes 71, San Salvador: IUDOP-UCA, $1998 b$.

Instituto Universitario de Opinión Pública (IUDOP), Encuesta sobre la gobernabilidad y expectativas hacia las nuevas autoridades municipales y legislativas, Serie de informes 64, San Salvador: IUDOPUCA, 1997.

Instituto Universitario de Opinión Pública (IUDOP), Encuesta sobre el sistema político salvadoreño, Serie de informes 51, San Salvador: IUDOP-UCA, 1995.

Instituto Universitario de Opinión Pública (IUDOP), "La opinión pública de los salvadoreños sobre las elecciones. La última encuesta pre-electoral", Estu- dios Centromericanos (ECA), 545-546, 1994, pp. 165-194.

Holston, James y Caldeira, Teresa, P.R., "Democracy, law, and violence: disjuctions of brazilian citizenship", en Felipe Agüero y. Jeffrey Stark (eds.), Fault lines of democracy in post-transition Latin America, Miami: North-South Center Press, 1998.

Inglehart, Ronald, "The renaissance of political culture", American Political Science Review, 82, 4, 1998, pp. 1203-1230.

Moreno-Martín, Florentino, Actitudes autoritarias y justificación de la violencia en Madrid, (Documento fotocopiado), 1997.

O’Donnell, Guillermo, “¿Democracia delegativa?”, en Jaime Barba (comp.), La democracia hoy, San Salvador: Istmo editores, 1994.

UNDP, Central America. Development in peace and democracy, San José: United Nations Develpment Program, 1998.

Valenzuela, J. Samuel, "Democratic consolidation in post-transitional settings: notion, process and facilitating conditions", en Scott Marinwaring, et. al., Issues in democratic consolidation, Notre Dame: University of Notre Dame Press, 1992. 